\title{
Skeletal myoblast transplantation for attenuation of hyperglycaemia, hyperinsulinaemia and glucose intolerance in a mouse model of type 2 diabetes mellitus
}

\author{
L. Ye • K. O. Lee $\cdot$ L. P. Su • W. C. Toh $\cdot$ H. K. Haider • \\ P. K. Law • W. Zhang • S. P. Chan • E. K. W. Sim
}

Received: 26 March 2009 / Accepted: 28 May 2009/Published online: 12 July 2009

(C) Springer-Verlag 2009

\begin{abstract}
Aims/hypothesis We aimed to demonstrate the feasibility and efficacy of intra-muscular transplantation of human skeletal myoblasts (hSkMs) for attenuation of hyperglycaemia and improvement of insulin sensitivity using a mouse model of type 2 diabetes mellitus.

Methods $\mathrm{KK} \mathrm{Cg-Ay/J} \mathrm{mice,} \mathrm{aged} 12$ to 14 weeks, underwent an initial intraperitoneal glucose tolerance test (GTT) and were divided into the following groups: KK control group, basal medium (M199) only; KK myoblast group, with hSkM transplantation; KK fibroblast group, with human fibroblast transplantation. Non-diabetic C57BL mice were used as an additional normal control and also
\end{abstract}

Electronic supplementary material The online version of this article (doi:10.1007/s00125-009-1421-9) contains supplementary material, which is available to authorised users.

L. Ye $\cdot$ W. C. Toh

National University Medical Institutes,

National University of Singapore,

Singapore, Republic of Singapore

K. O. Lee

Department of Medicine, National University of Singapore,

Singapore, Republic of Singapore

L. P. Su

Division of Bioengineering, National University of Singapore,

Singapore, Republic of Singapore

H. K. Haider

Department of Pathology and Lab Medicine,

University of Cincinnati,

Cincinnati, OH, USA

P. K. Law

Cell Transplants Singapore,

Singapore, Republic of Singapore had hSkM transplantation. Cells were transplanted intramuscularly into the skeletal muscles of the mice. All animals were treated with ciclosporin for 6 weeks only. $\mathrm{HbA}_{1 \mathrm{c}}$ and fasting GTT, as well as serum adiponectin, cholesterol, insulin and triacylglycerol were studied.

Results Immunohistochemistry studies showed extensive survival of the transplanted hSkMs in the skeletal muscles at 12 weeks, with nuclei of the hSkMs integrated into the host muscle fibres. Repeat GTT showed a significant decrease in glucose concentrations in the KK myoblast group compared with the KK control and KK fibroblast groups. The KK myoblast group also had reduced mean $\mathrm{HbA}_{1 \mathrm{c}}$, cholesterol, insulin and triacylglycerol, and increased adiponectin compared with the KK control and KK fibroblast groups. C57BL mice showed no change in glucose homeostasis after hSkM transplant.

\section{W. Zhang}

Department of Pharmacology, National University of Singapore, Singapore, Republic of Singapore

\section{S. P. Chan}

Business Analytics and Statistics, SIM University,

Singapore, Republic of Singapore

\section{E. K. W. Sim}

Gleneagles JPMC Cardiac Center, Jerudong, Brunei Darussalam

\section{E. K. W. $\operatorname{Sim}(\bowtie)$}

Mount Elizabeth Medical Centre,

3 Mount Elizabeth,

Singapore 228510, Republic of Singapore

e-mail: dr.eugene.sim@gmail.com 
Conclusions/interpretation Human skeletal myoblast transplantation attenuated hyperglycaemia and hyperinsulinaemia and improved glucose tolerance in the KK mouse. This novel approach of improving muscle insulin resistance may be a potential alternative treatment for type 2 diabetes mellitus.

Keywords Glucose tolerance · Hyperglycaemia - Insulin resistance $\cdot$ Skeletal muscle $\cdot$ Skeletal myoblasts .

Type 2 diabetes mellitus

$\begin{array}{ll}\text { Abbreviations } \\ \text { AKT } & \text { Protein kinase B } \\ \text { DMD } & \text { Duchenne muscular dystrophy } \\ \text { GTT } & \text { Glucose tolerance test } \\ \text { hSkM } & \text { Human skeletal myoblast } \\ \text { pAKT } & \text { Phosphorylated AKT } \\ \text { PI3K } & \text { p85a-Phosphoinositide 3-kinase }\end{array}$

\section{Introduction}

Type 2 diabetes mellitus is a major endocrine disease with a steadily increasing incidence [1], and an important cardiovascular disease risk factor [2, 3]. A key feature of type 2 diabetes mellitus is insulin resistance [4]. Epidemiological and twin studies have clearly indicated the importance of polygenetic as well as environmental factors in the development of insulin resistance [5-7].

Previous studies have demonstrated the importance of skeletal muscles in insulin resistance in type 2 diabetes mellitus. Mice with muscle-specific Glut4 knockout were insulinresistant and glucose-intolerant from an early age [8]. An isolated defect in protein kinase $\mathrm{C}-\lambda$ in muscle was sufficient to induce abdominal obesity and other metabolic abnormalities [9]. By contrast, muscle-specific LKB1 (a serine/threonine kinase that is a negative regulator of insulin sensitivity) knockout increased insulin sensitivity and improved glucose homeostasis [10]. These studies suggest that defects in skeletal muscle insulin-stimulated glucose transport may be key factors in insulin resistance and type 2 diabetes mellitus [11].

Using a novel cell therapy approach, we investigated whether skeletal muscle insulin resistance in type 2 diabetes mellitus can be corrected by xeno-transplantation of human skeletal myoblasts (hSkMs). Skeletal myoblasts are mononucleated precursor cells of skeletal muscle cells [12] that can fuse with different muscle fibre types and adopt their phenotype $[12,13]$. As skeletal muscles form approximately $30 \%$ to $50 \%$ of total body weight [14], we hypothesised that fusion of transplanted hSkMs from non-diabetic donors with host skeletal muscles of type 2 diabetes mellitus recipients could improve insulin sensitivity and glucose tolerance.

\section{Methods}

Animals and diets

All animals were maintained at the Animal Holding Unit of the National University of Singapore. All procedures were approved by the Institutional Animal Care and Use Committee.

KK mouse (Jackson Lab, Bar Harbor, ME, USA) is an animal model of type 2 diabetes mellitus, characterised by hyperglycaemia, hyperinsulinaemia and glucose intolerance [15]. C57BL mice were purchased from the University Animal Resources Centre. All mice were housed in individual cages at $25^{\circ} \mathrm{C}$ with a $12 \mathrm{~h}$ light-dark cycle and had free access to food (Lab Diet 5 K52; PMI Nutrition International, Brentwood, MO, USA) and water. Prior to study, KK mice were screened at 12 to 14 weeks of age for the following: (1) fasting blood glucose $>6.5 \mathrm{mmol} / \mathrm{l}$; (2) blood glucose $>20 \mathrm{mmol} / \mathrm{l}$ at 30 or $60 \mathrm{~min}$ and $>11 \mathrm{mmol} / \mathrm{l}$ at $2 \mathrm{~h}$ during glucose tolerance test (GTT).

\section{Glucose tolerance test}

All mice used in the study had a GTT after a 16-h overnight fast. Each mouse was injected intraperitoneally with $1 \mathrm{~g} / \mathrm{kg}$ body weight of glucose diluted in distilled water $(100 \mathrm{mg} / \mathrm{ml})$. Blood samples from the tail vein were taken at $0,0.5,1$ and $2 \mathrm{~h}$ after glucose injection. Blood glucose concentrations were determined by a glucometer (Accu-Chek Advantage; Roche Diagnostics, Mannheim Germany).

Fasting plasma metabolites

Blood samples were obtained from cavernous sinus sampling under anaesthesia. Plasma was stored at $-20^{\circ} \mathrm{C}$ until analysis. Plasma insulin concentrations were determined by an insulin ELISA kit (Mercodia, Uppsala, Sweden). Plasma cholesterol and triacylglycerol concentrations were determined using standard assays (Infinity Cholesterol; Thermo Electron, Thebarton, SA, Australia and Accutrend GCT, Roche). Adiponectin was determined by a mouse adiponectin ELISA Kit (Millipore, Billerica, MA, USA). $\mathrm{HbA}_{1 \mathrm{c}}$ was measured by an analyser (DCA 2000+; Bayer HealthCare, Elkhart, IN, USA).

\section{Culture and labelling of hSkMs}

Human skeletal myoblasts were purchased from Bioheart (Sunrise, FL, USA) and cultured in M199 medium supplemented with $10 \%$ fetal bovine serum (vol./vol.) and $10 \mathrm{ng} / \mathrm{ml}$ basic fibroblast growth factor (10\% M199) 
at $37^{\circ} \mathrm{C}$ in $5 \% \mathrm{CO}_{2}$ (vol./vol.) incubator. All hSkM cultures were assessed for the presence of desmin and CD56 as described earlier to control for purity [16]. Before transplantation, cells were dual-labelled with DAPI (Sigma, St Louis, MO, USA) and with BrdU using standard reagents (Roche) [17].

\section{Transplantation of hSkMs}

KK mice meeting the criteria were randomly assigned to two groups: KK control group $(n=16)$, receiving $1.5 \mathrm{ml}$ M199 medium; and KK myoblast group $1(n=16)$, receiving $1.5 \mathrm{ml}$ M199 medium containing $3 \times 10^{7} \mathrm{hSkMs}$. Non-diabetic C57BL mice served as an additional control group (C57BL myoblast group, $n=16$, receiving $1.5 \mathrm{ml}$ M199 medium containing $3 \times 10^{7} \mathrm{hSkMs}$ ). This group was used to assess for any adverse effects from hSkM transplantation.

After the initial series of experiments showed significant improvement in glucose and other variables, a second series of studies was conducted to determine whether the improvements in metabolic variables were a direct effect of hSkMs on muscle insulin resistance or attributable to the unexpected weight loss observed in the study group. In this second series, two groups of animals were compared: KK myoblast group $2(n=10)$, receiving $1.5 \mathrm{ml} \mathrm{M} 199$ medium containing $3 \times 10^{7} \mathrm{hSkMs}$; and KK fibroblast group $(n=10)$, receiving $1.5 \mathrm{ml}$ M199 basal medium containing $3 \times 10^{7}$ human fibroblasts (kind gift of T. T. Phan, Department of Surgery, National University of Singapore).

Cells were transplanted under anaesthesia by a number of direct injections into the hindlimb muscles, including the anteromedium muscles, posterior leg muscles and gluteal muscles. All animals were given ciclosporin $\left(10 \mathrm{mg} \mathrm{kg}^{-1}\right.$ day $^{-1}$ ) from 3 days before until 6 weeks after transplantation. All the animals were killed at 12 weeks.

Immunohistochemistry studies

The DAPI+ cryo-sections from mouse skeletal muscles removed at 12 weeks after cell transplantation underwent immunostaining for BrdU [17]. To visualise the integration of hSkM nuclei into mouse skeletal muscle fibres, DAPI+ cryo-sections also underwent immunofluorescent staining for determination of skeletal myosin and dystrophin levels [17].

Fluorescent in situ hybridisation analysis of mouse skeletal muscle

Cryo-sections ( $4 \mu \mathrm{m}$ thickness) of mouse skeletal muscle that had received hSkM transplantation (confirmed by DAPI expression) were used. The manufacturer's (Cambio, Cambridge, UK) protocol instructions were followed. The hybridisation probe mixture consisted of 1 $\mu 1$ human pan-centromeric probe conjugated with FITC (denatured at $85^{\circ} \mathrm{C}$ ) and $1 \mu \mathrm{l}$ mouse pan-centromeric conjugated with $\mathrm{Cy} 3$ (denatured at $60^{\circ} \mathrm{C}$ ) in $25 \mu \mathrm{l}$ of buffer. After chilling on ice, the probe mixture was applied on to the samples and covered with a glass cover slip, sealed and hybridised overnight at $37^{\circ} \mathrm{C}$. The next day, the samples were washed and dried in the dark and mounted with $10 \mu \mathrm{l}$ anti-fade solution containing $0.24 \mu \mathrm{g} / \mathrm{ml}$ DAPI for fluorescent microscopy.

\section{Western blot analysis}

At 12 weeks after transplantation, skeletal muscle tissues were used for western blot analysis and protein kinase B (AKT) and p85a-phosphoinositide 3-kinase (PI3K) activity assays. Half the mice from each group were killed after an overnight fast, while the remaining half were killed at $0.5 \mathrm{~h}$ after glucose i.p. injection. Total skeletal muscle protein was extracted using an extraction reagent (Phosphosafe; Merck, Darmstadt, Germany) and protein concentration was determined using Bradford reagent (Bio-Rad Laboratories, Hercules, CA, USA). Proteins ( $20 \mu \mathrm{g}$ per sample) were separated on SDS-polyacrylamide gel and transferred on to nitrocellulose membrane. The blots were incubated overnight with diluted 1:300 rabbit anti-insulin receptor, 1:300 antiIRS-1, 1:300 anti-phosphorylated (Tyr 632) IRS-1, 1:200 antiPI3K, 1:1,000 anti-AKT, 1:300 anti-phosphorylated (Ser 473) AKT (pAKT) and 1:300 anti-GLUT-4 (all from Santa Cruz Biotechnology, Santa Cruz, CA, USA) respectively. Antirabbit IgG conjugated with horseradish peroxidase (dilution: $1: 3,000-1: 8,000)$ was used as second antibody and the membrane was visualised by chemiluminescence (Thermo Scientific, Rockford, IL, USA) and exposed to x-ray film (Thermo Scientific). Optical density of the bands was quantified by Olympus Micro Image software (Olympus, Tokyo, Japan). The concentration of each protein sample was expressed as percentage of the $\mathrm{KK}$ control group or $\mathrm{KK}$ fibroblast group samples at baseline.

\section{Measurement of AKT activity}

AKT activity was measured using an assay for AKT/ PKB (Biosource Omnia Lysate, KNZ0011; Invitrogen, Carlsbad, CA USA) as per manufacturer's instructions. Mouse skeletal muscle tissue was lysed using Omnia cell extraction buffer and extracts containing $50 \mu \mathrm{g}$ total protein were used. The fluorescence intensity reading was measured every $1 \mathrm{~min}$ for $60 \mathrm{~min}$ using a microplate reader (Tecan Infinite M200; LabX, Midland, ON, Canada). The enzyme activity was expressed as: fluores- 
cence intensity $/ \mathrm{min}=($ end fluorescent intensity-start fluorescent intensity)/60 $\mathrm{min}$.

Measurement of PI3K activity

PI3K activity was quantified by a competition enzyme immunoassay kit according to the manufacturer's protocol (Echelon Bioscience, Salt Lake City, UT, USA). The enzyme activity was expressed as quantity of PIP3 (pmol mg ${ }^{-1} \mathrm{~h}^{-1}$ ) produced by $1 \mathrm{mg}$ of mouse skeletal muscle extracts containing equal amount of proteins in $1 \mathrm{~h}$ [18].

\section{Statistical analysis}

The data are presented as mean \pm SEM. Since GTT and the plasma insulin measurements were carried out repeatedly over time $(0,0.5,1.0$ and $2.0 \mathrm{~h})$, generalised estimating equations using Stata version 10.0 (StataCorp LP, College Station, TX, USA) with the linear link were applied to determine the difference between groups. The difference between the KK control group, KK myoblast group 1 and C57BL myoblast group was analysed by ANOVA with the Bonferroni test, while the difference between KK myoblast group 2 and the KK fibroblast group was analysed by independent $t$ tests.

\section{Results}

\section{Purity of hSkM culture}

All hSkM cultures had greater than 90\% purity as assessed by desmin or CD56 (Fig. 1a-d) and by flow cytometry (Fig. 1e-g).

Improvement of fasting blood metabolites

Glucose tolerance tests Intraperitoneal GTT demonstrated that KK mice developed hyperglycaemia and glucose intolerance at 12 to 14 weeks of age (Fig. 2a). Glucose
Fig. 1 Characterisation of hSkMs for abundance of desmin and CD56. Dual fluorescent immunostaining of hSkMs for (a) desmin and (b) CD56. c Superimposition of above $(\mathbf{a}, \mathbf{b})$ to show desmin and CD56 simultaneously using (d) fibroblasts as negative control Green fluorescence, desmin; red fluorescence, CD56. Magnification $200 \times$. e Flow cytometry analysis of hSkMs for desmin and CD56 (91.9\% of cells positive for desmin +CD56), using (f) non-stained hSkMs as baseline for auto-fluorescence $(0.8 \%$ of cells positive) and (g) fibroblasts as negative control ( $2.6 \%$ of cells positive)
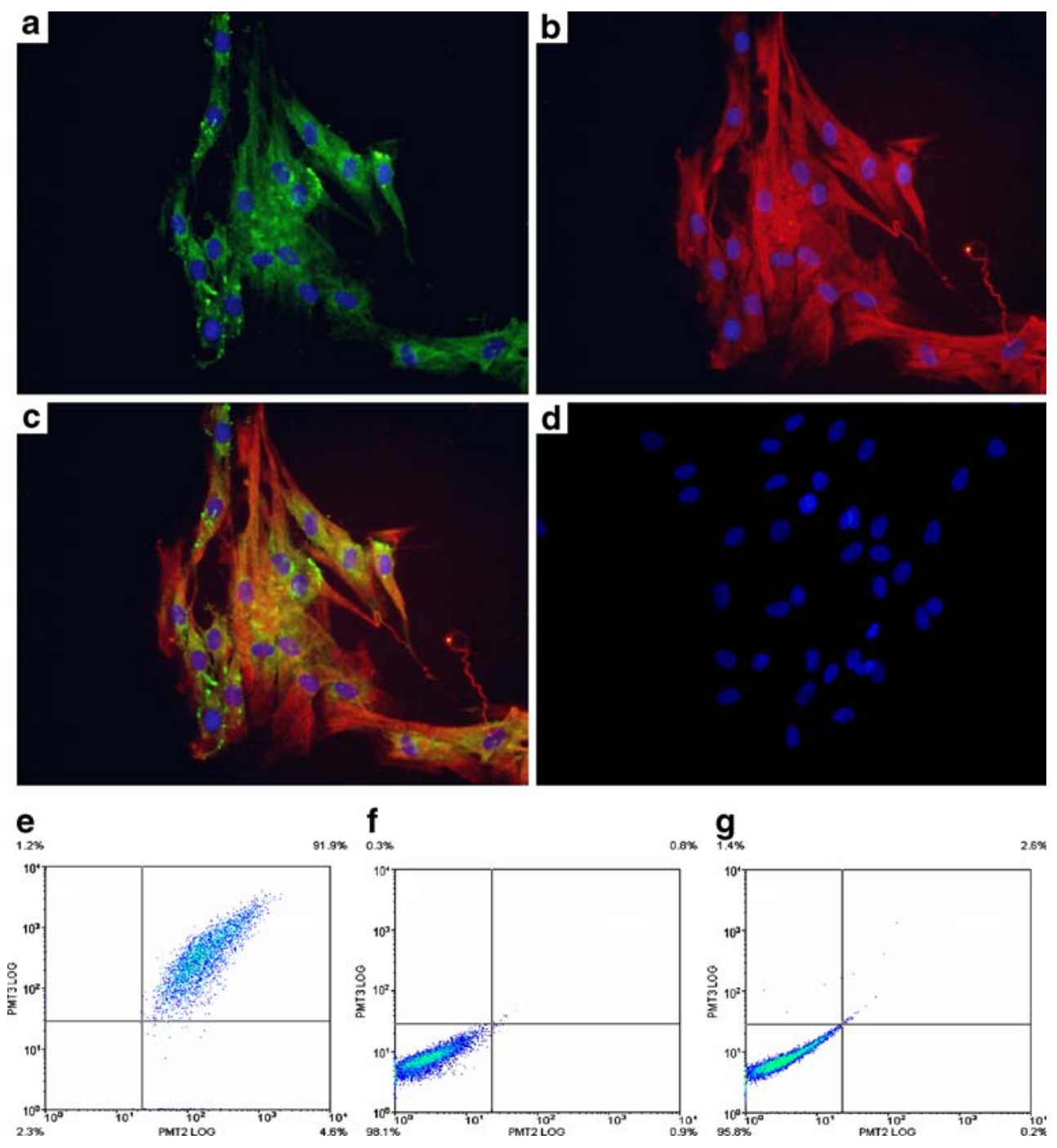

f

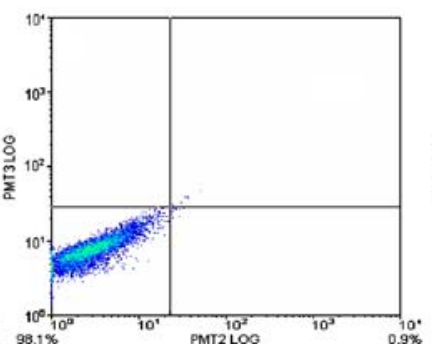

g

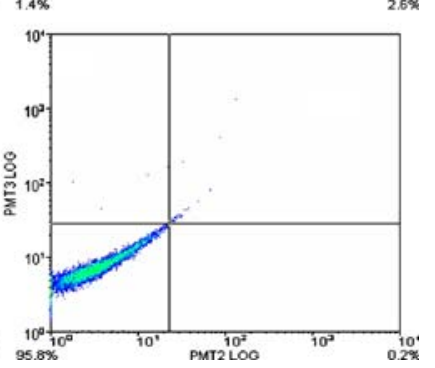


a

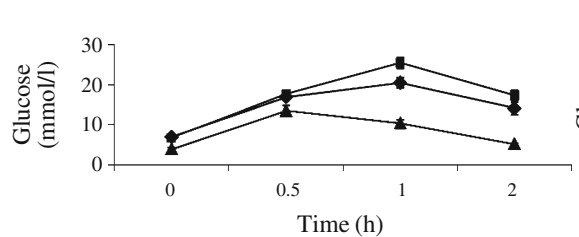

d
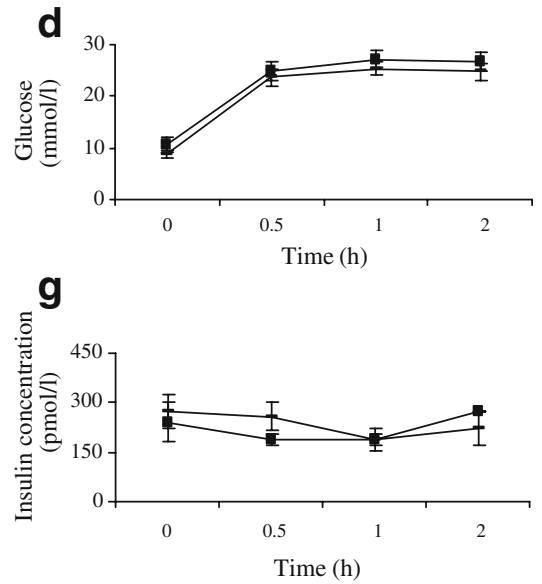

b

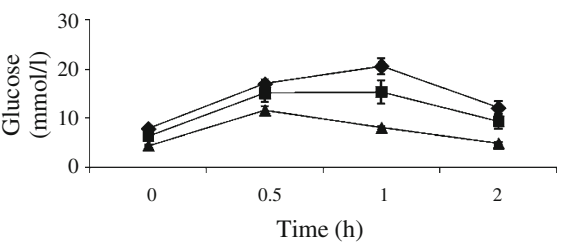

e
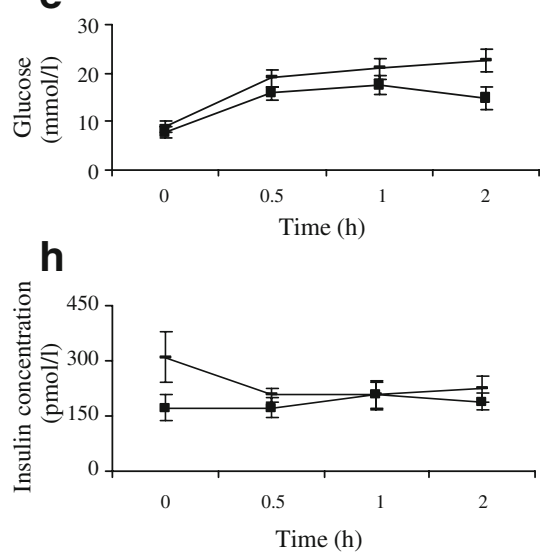

C

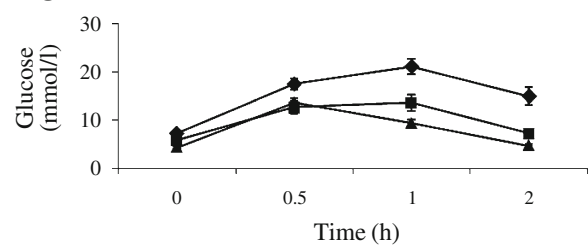

f
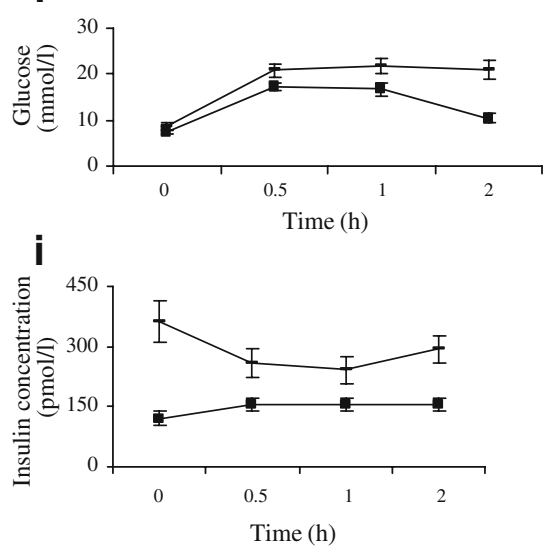

Fig. 2 Glucose tolerance tests of the KK control group (diamonds), KK myoblast group 1 (squares) and C57BL myoblast group (triangles) at (a) baseline, (b) 6 weeks and (c) 12 weeks after treatment. Glucose tolerance tests of the KK myoblast group 2 (squares) and the KK

fibroblast group (rectangles) at (d) baseline, (e) 6 weeks and (f) 12 weeks after treatment. $\mathbf{g}-\mathbf{i}$ Plasma insulin of the KK myoblast group 2 and the KK fibroblast group during glucose tolerance tests at (g) baseline, (h) 6 weeks and (i) 12 weeks after treatment

concentrations $(\mathrm{mmol} / \mathrm{l})$ of all $\mathrm{KK}$ groups were significantly higher than those in the C56BL myoblast group $(p<0.05)$.

At 6 weeks after transplantation, KK control group animals remained hyperglycaemic and glucose-intolerant (Fig. 2b). Blood glucose was reduced (but not statistically significant) in the $\mathrm{KK}$ myoblast group 1 and remained normal in the C57BL myoblast group $(p<0.05)$.

At 12 weeks after transplantation, KK control group animals still had severe hyperglycaemia and glucose intolerance (Fig. 2c). By contrast, KK myoblast group 1 animals showed a significant reduction in blood glucose $(p<0.05)$, while that in the C57BL myoblast group remained normal $(p<0.05)$.

These differences in glucose concentrations were similarly seen in the second series of experiments comparing KK myoblast group 2 with the KK fibroblast group (Fig. $2 \mathrm{~d}-\mathrm{f}$ ) $(p<0.05$ for 6 and 12 weeks after cell transplantation).

Plasma insulin during GTT Plasma insulin was not measured in the first series of experiments, but in the second series it was found to be decreased in KK myoblast group 2 mice when compared with the KK fibroblast group (Fig. 2g-i). At 12 weeks, plasma insulin was significantly lower than in the KK fibroblast group during GTT $(p<0.05)$.

Other plasma metabolites The results of the fasting plasma metabolites are shown in Tables 1 and 2. Fasting cholesterol, insulin and triacylglycerol in the KK control group and KK myoblast group 1 were significantly higher (increased more than 64, 145 and $67 \%$, respectively) than those of the C57BL myoblast group at 12 to 14 weeks of age. At 6 weeks after treatment, fasting cholesterol, insulin and triacylglycerol were still higher in the $\mathrm{KK}$ control group and $\mathrm{KK}$ myoblast group 1 than in the $\mathrm{C} 57 \mathrm{BL}$ myoblast group. At 12 weeks after treatment, the KK control group had further increases in fasting insulin, cholesterol and triacylglycerol, while the KK myoblast group 1 had significant reductions of fasting cholesterol, insulin and triacylglycerol $(p<0.05)$.

At 12 to 14 weeks of age, KK mice had significantly lower serum adiponectin than C57BL mice. Serum adiponectin continued to decrease in the KK control group, but not in KK myoblast group 1 at 6 and 12 weeks after transplantation. These data suggest that adiponectin may be involved in mediating the effects of hSkM transplantation. In the second series of experiments, the KK myoblast group 2 mice had a similarly significantly higher adiponectin than the $\mathrm{KK}$ fibroblast group at 12 weeks after transplantation (Table 2).

Reduced blood $\mathrm{HbA}_{1 \mathrm{c}}$

Glycosylated haemoglobin demonstrated that the KK myoblast group mice achieved significantly better glucose 
Table 1 Body weight and plasma metabolites of the KK control group, KK myoblast group 1 and C57BL myoblast group at baseline and after treatment as shown

\begin{tabular}{|c|c|c|c|}
\hline \multirow[t]{2}{*}{ Variables per status } & \multicolumn{3}{|l|}{ Animal groups } \\
\hline & KK DMEM group $(n=16)$ & KK myoblast $1(n=16)$ & C57BL myoblast group $(n=16)$ \\
\hline \multicolumn{4}{|l|}{ Baseline } \\
\hline Weight (g) & $28.89 \pm 1.37$ & $28.33 \pm 0.82$ & $21.13 \pm 0.41^{*}$ \\
\hline Adiponectin $(\mu \mathrm{g} / \mathrm{ml})$ & $4.57 \pm 0.33$ & $4.65 \pm 0.36$ & $7.25 \pm 0.57^{*}$ \\
\hline Cholesterol (mmol/l) & $3.66 \pm 0.34$ & $3.84 \pm 0.35$ & $2.23 \pm 0.1^{*}$ \\
\hline Fasting insulin (pmol/1) & $132.6 \pm 27.5$ & $130.9 \pm 15.5$ & $53.4 \pm 3.4 *$ \\
\hline Triacylglycerol (mmol/l) & $2.2 \pm 0.09$ & $2.32 \pm 0.19$ & $1.32 \pm 0.09^{*}$ \\
\hline $\mathrm{HbA}_{1 \mathrm{c}}(\%)$ & $4.64 \pm 0.14$ & $4.92 \pm 0.23$ & $3.83 \pm 0.12 *$ \\
\hline \multicolumn{4}{|l|}{6 weeks after treatment } \\
\hline Weight (g) & $32 \pm 1.62$ & $29.36 \pm 1.43$ & $21.75 \pm 0.68^{*}$ \\
\hline Adiponectin $(\mu \mathrm{g} / \mathrm{ml})$ & $4.32 \pm 0.33$ & $4.61 \pm 0.21$ & $8.36 \pm 0.38^{*}$ \\
\hline Cholesterol (mmol/l) & $3.85 \pm 0.19$ & $3.64 \pm 0.29$ & $2.55 \pm 0.13^{*}$ \\
\hline Fasting insulin (pmol/1) & $149.8 \pm 27.5$ & $122.2 \pm 13.8$ & $51.7 \pm 3.4^{*}$ \\
\hline Triacylglycerol (mmol/1) & $2.29 \pm 0.11$ & $1.92 \pm 0.09$ & $1.32 \pm 0.05^{*}$ \\
\hline $\mathrm{HbA}_{1 \mathrm{c}}(\%)$ & NA & NA & NA \\
\hline \multicolumn{4}{|l|}{12 weeks after treatment } \\
\hline Weight (g) & $34 \pm 1.44$ & $28.54 \pm 1.15^{\dagger}$ & $21.6 \pm 0.6^{*}$ \\
\hline Adiponectin $(\mu \mathrm{g} / \mathrm{ml})$ & $3.43 \pm 0.22$ & $4.84 \pm 0.33^{\dagger}$ & $7.98 \pm 0.3 *$ \\
\hline Cholesterol (mmol/l) & $3.8 \pm 0.24$ & $3.47 \pm 0.22$ & $2.48 \pm 0.09^{*}$ \\
\hline Fasting insulin (pmol/l) & $204.9 \pm 27.5$ & $99.9 \pm 17.2^{\dagger}$ & $55.1 \pm 5.2^{\dagger}$ \\
\hline Triacylglycerol (mmol/l) & $2.26 \pm 0.16$ & $1.7 \pm 0.13^{\dagger}$ & $1.19 \pm 0.03^{\dagger}$ \\
\hline $\mathrm{HbA}_{1 \mathrm{c}}(\%)$ & $6.0 \pm 0.11$ & $4.3 \pm 0.19^{\dagger}$ & $3.81 \pm 0.08^{\dagger}$ \\
\hline
\end{tabular}

Values are presented as mean $\pm \mathrm{SEM}$

${ }^{*} p<0.05$ vs $\mathrm{KK}$ control group and $\mathrm{KK}$ myoblast group $1 ;{ }^{\dagger} p<0.05$ vs $\mathrm{KK}$ control group

NA, not applicable

control after hSkM transplantation (Tables 1 and 2). Before treatment, $\mathrm{HbA}_{1 \mathrm{c}}$ was higher in the $\mathrm{KK}$ control and $\mathrm{KK}$ myoblast group 1 than in the C57BL myoblast group ( $p<$ 0.05 ) (Table 1). At 12 weeks after treatment, $\mathrm{HbA}_{1 \mathrm{c}}$ in $\mathrm{KK}$ control group further increased compared with the transplanted KK myoblast group $(p<0.05)$ and that of C57BL myoblast group $(p<0.05)$. In the second series of experiments, $\mathrm{HbA}_{1 \mathrm{c}}$ in the $\mathrm{KK}$ myoblast group 2 and $\mathrm{KK}$ fibroblast group also indicated better control of glucose metabolism after hSkM transplantation compared with fibroblast transplantation.

Body weight change

The body weights of the KK control group increased by $17.7 \%$ at 12 weeks after M199 medium injection, while it remained unchanged in KK myoblast and $\mathrm{KK}$ fibroblast groups during the experiment (Tables 1 and 2). This led to a suggestion that the change in body weight could have contributed to the improvement in glucose metabolism. The second series of experiments indicated that glucose control and metabolism improved even when the body weights of both transplanted groups did not differ.

Survival and integration of hSkMs into host mouse skeletal muscle

The labelling efficiency of hSkMs was $100 \%$ for DAPI and $50 \%$ to $60 \%$ for BrdU (Electronic supplementary material [ESM] Fig. 1a, b). Extensive survival of hSkMs shown as DAPI + or BrdU+ nuclei was found in mouse skeletal muscles at 12 weeks after cell transplantation (ESM Fig. 1c-f). By contrast, almost no DAPI+ fibroblast nuclei were found in the KK fibroblast group mice at 12 weeks after transplantation. The survival of hSkMs was further confirmed by fluorescent in situ hybridisation analysis showing that human and mouse pan-centromeric probe-labelled nuclei co-localised in the same tissue section (Fig. 3a, b, ESM Fig. 1g-j).

Immunostaining of DAPI+ tissue for skeletal myosin showed that hSkMs nuclei were co-localised with host nuclei in the same muscle fibres, demonstrating that nuclei of hSkMs had integrated into host skeletal muscle fibres to 
Table 2 Body weight and plasma metabolites of KK myoblast group 2 and the KK fibroblast group at baseline and after treatment as shown

Values are presented as mean \pm SEM

${ }^{*} p<0.05$ vs KK fibroblast group NA, not applicable

\begin{tabular}{|c|c|c|}
\hline \multirow[t]{2}{*}{ Variables per status } & \multicolumn{2}{|l|}{ Animal group } \\
\hline & KK myoblast group $2(n=10)$ & KK fibroblast group $(n=10)$ \\
\hline \multicolumn{3}{|l|}{ Baseline } \\
\hline Weight (g) & $32.7 \pm 0.8$ & $32.7 \pm 1.0$ \\
\hline Adiponectin $(\mu \mathrm{g} / \mathrm{ml})$ & $4.0 \pm 0.5$ & $3.8 \pm 0.3$ \\
\hline Cholesterol (mmol/l) & $4.1 \pm 0.2$ & $3.9 \pm 0.2$ \\
\hline Fasting insulin (pmol/1) & $241 \pm 51.7$ & $275.5 \pm 51.7$ \\
\hline Triacylglycerol (mmol/l) & $3.2 \pm 0.1$ & $3.4 \pm 0.1$ \\
\hline $\mathrm{HbA}_{1 \mathrm{c}}(\%)$ & $6.4 \pm 0.3$ & $6.3 \pm 0.3$ \\
\hline \multicolumn{3}{|l|}{6 weeks after treatment } \\
\hline Weight (g) & $32.3 \pm 0.8$ & $31.1 \pm 1.1$ \\
\hline Adiponectin $(\mu \mathrm{g} / \mathrm{ml})$ & $4.6 \pm 0.6$ & $3.9 \pm 0.2$ \\
\hline Cholesterol (mmol/l) & $4.0 \pm 0.1$ & $4.6 \pm 0.4$ \\
\hline Fasting insulin (pmol/1) & $172.2 \pm 17.2$ & $310 \pm 68.9$ \\
\hline Triacylglycerol (mmol/l) & $2.9 \pm 0.1$ & $3.4 \pm 0.3$ \\
\hline $\mathrm{HbA}_{1 \mathrm{c}}(\%)$ & NA & NA \\
\hline \multicolumn{3}{|l|}{12 weeks after treatment } \\
\hline Weight (g) & $32.5 \pm 1.0$ & $32.2 \pm 1.4$ \\
\hline Adiponectin $(\mu \mathrm{g} / \mathrm{ml})$ & $4.7 \pm 0.3^{*}$ & $3.5 \pm 0.2$ \\
\hline Cholesterol (mmol/l) & $3.8 \pm 0.1 *$ & $4.7 \pm 0.2$ \\
\hline Fasting insulin (pmol/1) & $120.5 \pm 17.2^{*}$ & $361.6 \pm 74.0$ \\
\hline Triacylglycerol (mmol/l) & $2.7 \pm 0.2^{*}$ & $3.4 \pm 0.2$ \\
\hline $\mathrm{HbA}_{1 \mathrm{c}}(\%)$ & $5.2 \pm 0.1 *$ & $6.5 \pm 0.4$ \\
\hline
\end{tabular}

form hybrid muscle fibres (Fig. 4a, ESM Fig. 2a, b). This was further confirmed by immunostaining for dystrophin (Fig. 4b, ESM Fig. 2c, d).

Protein level of insulin signalling pathway

At 12 weeks after transplantation, western blot showed a decrease in levels of insulin receptor, IRS-1, PI3K, AKT and Glut4 in all KK animal groups at baseline condition compared with C57BL mice (ESM Fig. 3). Glucose stimulation did not significantly change the levels of these proteins. However, phosphorylated IRS-1 and pAKT were
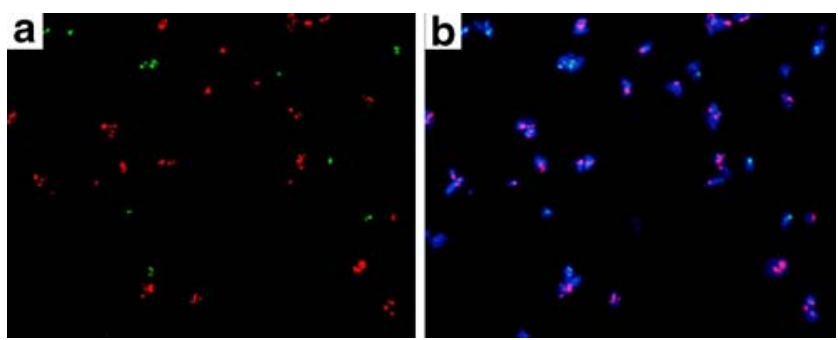

Fig. 3 a Human and mouse pan-centromeric probe-labelled nuclei (green and red fluorescence, respectively) were co-localised in the same tissue section (b) that was counterstained with DAPI (blue fluorescence) to show all nuclei. Magnification 400× significantly increased in KK myoblast mice compared with KK controls and/or KK fibroblast mice after glucose stimulation (Fig. 5). These results suggest that intramuscular transplantation of hSkMs increased phosphorylated IRS-1 and pAKT activities in response to glucose stimulation, which would then lead to increased uptake of glucose by skeletal muscles.

\section{Activity of AKT and PI3K}

At 12 weeks after transplantation, AKT activity (fluorescence intensity/min) of the KK control group at basal condition was similar to that of the KK myoblast group $1(p>0.05)$ and lower than that of the C57BL myoblast group $(p<0.05)$ (ESM Fig. 4a). After stimulation by i.p. glucose injection, AKT activity increased to $74.08 \pm 6.08$ in the KK myoblast group 1, which is significantly higher than 48.24 7.1 in $\mathrm{KK}$ control group $(p<0.05)$, but similar to the C57BL myoblast group (78.6 \pm 5.14$)$ (ESM Fig. 4b).

At 12 weeks after transplantation, PI3K activity ( $\mathrm{pmol} \mathrm{mg}^{-1} \mathrm{~h}^{-1}$ ) of all three groups, i.e. the KK control group, KK myoblast group 1 and C57Bl myoblast group, at basal condition was similar $(p>0.05)$ (ESM Fig. 4c). After i.p. glucose stimulation, PI3K activity was almost unchanged in the KK control group (105.19 \pm 10.39$)$, but increased significantly to $154.26 \pm 8.66(p<0.05)$ in the 

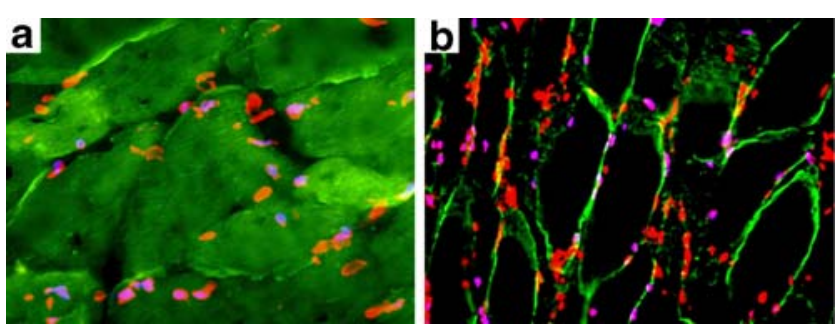

Fig. 4 Integration of hSkM nuclei into mouse skeletal muscle fibres. a Co-localisation of DAPI+ hSkM nuclei with mouse nuclei in the same muscle fibre after imuunostaining for skeletal myosin (green fluorescence). b Co-localisation of DAPI+ hSkM nuclei with mouse nuclei in the same muscle fibre after immunostaining for dystrophin (green fluorescence) to delineate the boundary of skeletal myofibres. Blue fluorescence, DAPI $+\mathrm{hSkMs}$; orange fluorescence, iodine staining to show all the nuclei. Magnification (a) $400 \times$, (b) $200 \times$

KK myoblast group 1 and also in the C57BL group, $173.17 \pm 18.41(p<0.05)$ (ESM Fig. 4d).

\section{Discussion}

We have demonstrated that hSkM transplantation into limb skeletal muscles of the KK mouse animal model of type 2 diabetes mellitus can ameliorate hyperglycaemia and hyperinsulinaemia, and improve glucose tolerance. We have further demonstrated that donor hSkM can integrate into host mouse skeletal muscles and lead to improved PI3K and AKT activity. This novel approach may be potentially useful in reversing muscle insulin resistance in patients with type 2 diabetes mellitus.

Decreased insulin-induced glucose uptake in skeletal muscle plays an important role in development of type 2 diabetes mellitus $[19,20]$. Skeletal muscle is a long-living tissue that makes up $30 \%$ to $50 \%$ of total human body mass [14] and is responsible for about $75 \%$ of whole-body glucose metabolism [21]. Skeletal muscle myofibres are formed by the fusion of multiple mononucleated skeletal myoblasts $[22,23]$. They are able to continually fuse with neighbouring mature myofibres throughout life, aiding regeneration after injury to muscle tissue [12]. The nucleus of skeletal myoblast from a normal donor contains more than 30,000 normal genes that determine its normality and cell characteristics [22]. It is possible that fusion between donor skeletal myoblasts and host skeletal muscle fibres might enable the donor nuclei to supplement defective genes involved in insulin-stimulated glucose transport into skeletal muscle, thus correcting defects that lead to insulin resistance.

Our present study demonstrates that this novel approach led to significant reduction in hyperglycaemia, hyperinsulinaemia, cholesterol, triacylglycerol and $\mathrm{HbA}_{1 \mathrm{c}}$, and an increase in adiponectin in $\mathrm{KK}$ animals after $\mathrm{hSkM}$ transplantation. The maximal benefit was achieved at 12 weeks after treatment. The body weight of the KK control group continued to increase by $17.7 \%$ at 12 weeks after treatment, while it remained unchanged in KK myoblast
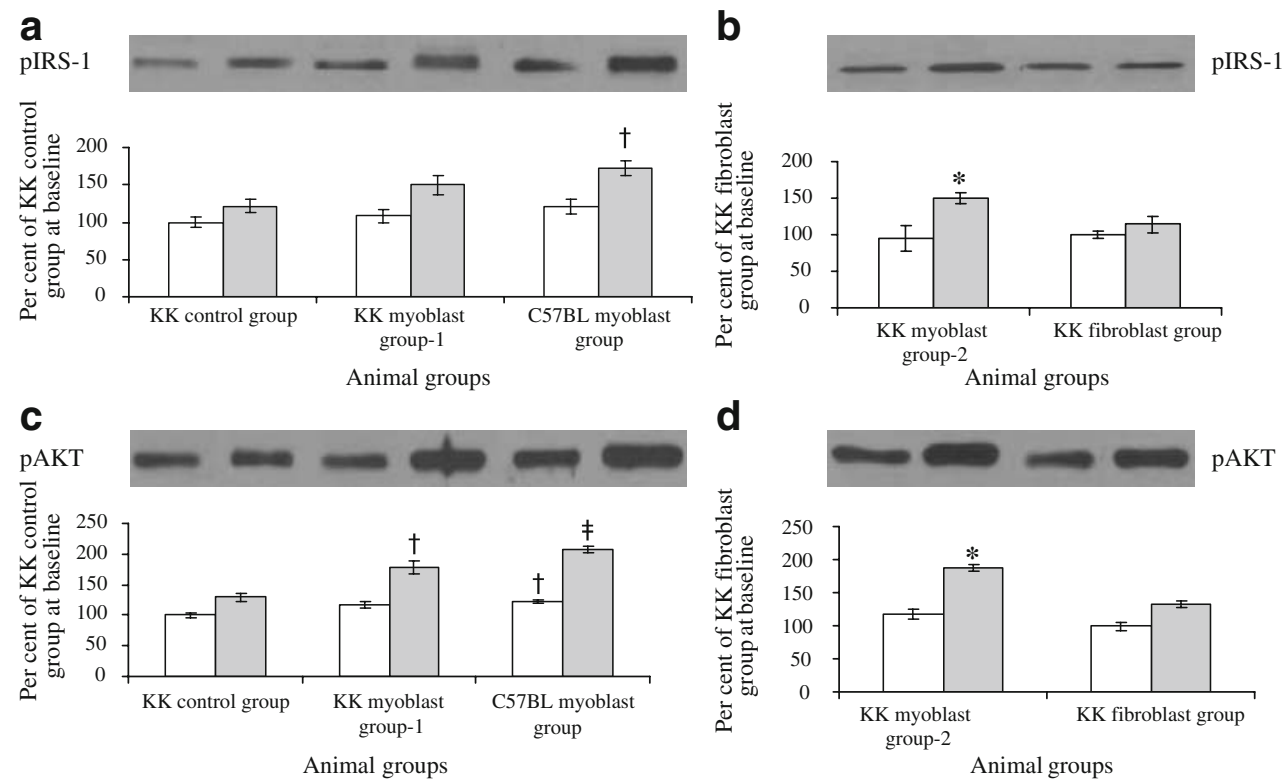

Fig. 5 Western blot analyses (gels and bar graphs) for protein levels of pIRS- 1 at basal condition and $0.5 \mathrm{~h}$ after i.p. glucose injection in (a) KK control, KK myoblast 1, and C57BL myoblast groups, and (b) KK myoblast 2 and $\mathrm{KK}$ fibroblast groups. Western blot analyses (gels and bar graphs) for protein levels of pAKT at basal condition and $0.5 \mathrm{~h}$ after

i.p. glucose injection in (c) KK control, KK myoblast 1, and C57BL myoblast groups, and (d) KK myoblast 2 and $\mathrm{KK}$ fibroblast groups. White bar, basal condition; grey bar, $0.5 \mathrm{~h}$ after i.p. glucose injection. ${ }^{\dagger} p$ $<0.05$ vs KK control group; ${ }^{\ddagger} p<0.05$ vs KK control group and KK myoblast group-1; ${ }^{*} p<0.05$ vs KK fibroblast group 
and KK fibroblast groups. One possible reason may be the immunological response to cell transplantation causing a change in body weight in the transplanted groups compared with the control group. Immunosuppression was used in all groups for only 6 weeks. This would allow hSkMs to escape the initial host immune rejection and allow fusion with host muscle fibres. The unfused hSkMs and fibroblasts would be eliminated by host immune system at 12 weeks after transplantation. Our immunohistochemistry data demonstrate that hSkMs survived extensively in mouse skeletal muscle at 12 weeks after cell transplantation. hSkM nuclei integrated into the host skeletal muscle fibres to form hybrid muscle fibres. Once fusion has occurred, the donor hSkMs will escape host immuno-rejection despite withdrawal of ciclosporin treatment, as mature skeletal muscle fibres do not express major histocompatibility complex class I antigens [24]. The donor myoblast nuclei will probably continue to co-express other genes together with mouse skeletal muscle genes in the hybrid muscle fibres. This has been demonstrated in skeletal myoblast transplantation for the treatment of Duchenne's muscular dystrophy (DMD), where implantation/transplantation of normal skeletal myoblasts would alleviate muscle fibre degeneration by producing an intact dystrophin within the host muscles $[25,26]$.

Our western blots showed that hSkM transplantation improved activities of phosphorylated IRS-1 and pAKT compared with the KK control group. Our kinase activity assay showed that the baseline activities of PI3K and AKT in $\mathrm{KK}$ myoblast group 1 were increased by $11.7 \%$ and $25.5 \%$, respectively, compared with the KK control group. These further increased by $31.26 \%$ and $147.67 \%$ in KK myoblast group 1 after i.p. glucose injection. These data support the hypothesis that hSkM transplantation increased activity of AKT and PI3K in KK mouse muscle fibres. The increased phosphorylation at any of several possible sites on these proteins could potentially increase their ability to bind and activate various downstream effectors in the insulin-mediated signal transduction pathway in glucose transportation into skeletal muscle [27].

The basis of our cell therapy study for insulin resistance is similar to that used for the treatment of DMD [28-31]. However, DMD is a single gene defect with complete absence of production of a single protein, whereas type 2 diabetes is a polygeneic disease with potentially decreased levels of multiple proteins. The reversal of DMD may thus require a much higher level of dystrophin and supplementation of dystrophin by hSkM at a low level may be insufficient to completely reverse this defect. Insulin resistance, being polygeneic in aetiology, might be improved by supplementing multiple genes and proteins involved in insulin-mediated glucose transport and metabolism.
In conclusion, our data indicate that xeno-transplantation of normal hSkMs into limb skeletal muscles of diabetic KK mice may be useful as a novel approach to treatment of type 2 diabetes mellitus, specifically targeting muscle insulin resistance. Future studies to explore the underlying molecular bases and mechanisms are needed and may give new insights to skeletal muscle insulin resistance.

Acknowledgements The project was funded by a block grant of the NMRC Singapore awarded to the National University Medical Institutes, NUS, Singapore (R-364-000-021-213).

Duality of interest The authors declare that there is no duality of interest associated with this manuscript.

\section{References}

1. Mokdad AH, Ford ES, Bowman BA et al (2001) The continuing increase of diabetes in the US. Diabetes Care 24:412

2. Haffner SM, Lehto S, Ronnemaa T, Pyorala K, Laakso M (1998) Mortality from coronary heart disease in subjects with type 2 diabetes and in nondiabetic subjects with and without prior myocardial infarction. N Engl J Med 339:229-234

3. Krone W, Meinertz T (1995) Metabolic syndrome as a cardiovascular risk factor. Herz 20:2-4 (article in German)

4. Baudler S, Krone W, Brüning JC (2003) Genetic manipulation of the insulin signalling cascade in mice - potential insight into the pathomechanism of type 2 diabetes. Best Pract Res Clin Endocrinol Metab 17:431-443

5. Guillausseau PJ, Tielmans D, Virally-Monod M, Assayag M (1997) Diabetes: from phenotypes to genotypes. Diabetes Metab 23:14-21

6. Medici F, Hawa M, Ianari A, Pyke DA, Leslie RD (1999) Concordance rate for type 2 diabetes mellitus in monozygotic twins: actuarial analysis. Diabetologia 42:146-150

7. Stern MP (1999) Genetic and environmental influences on type 2 diabetes mellitus in Mexican Americans. Nutr Rev 57:S66-S70

8. Zisman A, Peroni OD, Abel ED et al (2000) Targeted disruption of the glucose transporter 4 selectively in muscle causes insulin resistance and glucose intolerance. Nat Med 6:924-928

9. Farese RV, Sajan MP, Yang H et al (2007) Muscle-specific knockout of PKC- $\lambda$ impairs glucose transport and induces metabolic and diabetic syndromes. J Clin Invest 117:2289-2301

10. Koh HJ, Arnolds DE, Fujii N et al (2006) Skeletal muscle-selective knockout of LKB1 increases insulin sensitivity, improves glucose homeostasis, and decreases TRB3. Mol Cell Biol 26:8217-8227

11. Saltiel AR (2001) New perspectives into the molecular pathogenesis and treatment of type 2 diabetes. Cell 104:517-529

12. Mauro A (1961) Satellite cell of skeletal muscle fibers. J Biophys Biochem Cytol 9:493-495

13. Campion DR (1984) The muscle satellite cell: a review. Int Rev Cytol 87:225-251

14. Kim J, Wang ZM, Heymsfield SB, Baumgartner RN, Gallagher D (2002) Total-body skeletal muscle mass: estimation by a new dual-energy X-ray absorptiometry method. Am J Clin Nutr 76:378-383

15. Iwatsuka H, Shino A, Suzuoki Z (1970) General survey of diabetic features of yellow KK mice. Endocrinol Jpn 17:23-35

16. Ye L, Haider Kh H, Tan RS et al (2007) Transplantation of nanoparticle transfected skeletal myoblasts over-expressing vascular endothelial growth factor-165 for cardiac repair. Circulation 116:I113-I120 
17. Ye L, Haider Kh H, Tan RS et al (2008) Angiomyogenesis using liposome based vascular endothelial growth factor-165 transfection with skeletal myoblast for cardiac repair. Biomaterials 29:2125-2137

18. Lee KS, Kim SR, Park SJ et al (2006) Phosphatase and tensin homolog deleted on chromosome 10 (PTEN) reduces vascular endothelial growth factor expression in allergen-induced airway inflammation. Mol Pharmacol 69:1829-1839

19. Reaven GM (1995) Pathophysiology of insulin resistance in human disease. Physiol Rev 75:473-486

20. Ferrannini E (1997) Insulin resistance is central to the burden of diabetes. Diabetes Metab Rev 13:81-86

21. Zierath JR, Krook A, Wallberg-Henriksson H (2000) Insulin action and insulin resistance in human skeletal muscle. Diabetologia 43:821-835

22. Law PK, Goodwin TG, Fang Q et al (1997) First human myoblast transfer therapy continues to show dystrophin after 6 years. Cell Transplant 6:95-100

23. Bischoff R, Heintz C (1994) Enhancement of skeletal muscle regeneration. Dev Dyn 201:41-54

24. Karpati G, Pouliot Y, Carpenter S (1988) Expression of immunoreactive major histocompatibility complex products in human skeletal muscles. Ann Neurol 23:64-72
25. Partridge TA, Morgan JE, Coulton GR, Hoffman EP, Kunkel LM (1989) Conversion of $\mathrm{mdx}$ myofibres from dystrophin-negative to -positive by injection of normal myoblasts. Nature 337:176179

26. Morgan JE, Hoffman EP, Partridge TA (1990) Normal myogenic cells from newborn mice restore normal histology to degenerating muscles of the $\mathrm{mdx}$ mouse. J Cell Biol 111:2437-2449

27. Morris F, White C, Ronald K (1994) Insulin signaling system. J Biol Chem 269:1-4

28. Watt DJ, Morgan JE, Partridge TA (1984) Use of mononuclear precursor cells to insert allogeneic genes into growing mouse muscles. Muscle Nerve 7:741-750

29. Watt DJ, Morgan JE, Clifford MA, Partridge TA (1987) The movement of muscle precursor cells between adjacent regenerating muscles in the mouse. Anat Embryol 175:527-536

30. Gussoni E, Wang Y, Fraefel C et al (1996) A method to codetect introduced genes and their products in gene therapy protocols. Nat Biotechnol 14:1012-1016

31. Gussoni E, Blau HM, Kunkel LM (1997) The fate of individual myoblasts after transplantation into muscles of DMD patients. Nat Med 3:970-977 